\title{
Collaborative Exchange of Cargo Truck loads: Approaches to reducing empty trucks in logistics chains
}

\author{
Hans-Henrik Hvolby ${ }^{1(\bowtie)}[0000-0002-5574-5216]$ Kenn Steger-Jensen ${ }^{1}$, Mihai Neagoe 2 [0000- \\ 0003-4362-8132] Sven Vestergaard ${ }^{1 \text { [to come] }}$ and Paul Turner ${ }^{2}$ [0000-0003-4504-2338] \\ ${ }^{1}$ Centre for Logistics, Department of Materials \& Production, \\ Aalborg University, Denmark \\ $\bowtie$ hhh@celog.dk
}

${ }^{2}$ ARC Centre for Forest Value, Discipline of ICT, College of Sciences and Engineering, University of Tasmania, Hobart, Australia

\begin{abstract}
Reducing the volume of trucks carrying empty or below capacity loads on road networks are both socio-economic and environmental sustainability issues for the logistics industry. Planning concepts for a collaborative logistics exchange based on real-time data are described as well as the benefits in terms of optimizing load capacity utilization, minimization of empty running, reducing costs, traffic congestion, and truck emissions
\end{abstract}

Keywords: Collaborative, transport, cargo, logistics, agent-based, constraints, optimise

\section{Introduction.}

Based on data from the European Union [1] identified that empty backhauls represent about $25 \%$ of the road transportation activities and that loaded trucks in average use $57 \%$ of the capacity. This triggered a further investigation into how planning of truckloads is conducted as well as conditions and rules for transport companies. This research project was initiated with the aim to reduce the empty running by exchanging loads among freight carriers. The centre-point of the project is to provide real-time data and advanced decision support tools to the transport companies and thereby reduce costs as well as environmental benefits in terms of reduced pollution and congestion.

The potential benefits of horizontal collaboration for logistics services providers are recognized in the literature in the form of increased efficiency and productivity gains [2], decreased environmental impact, and improved market presence or access [3, 4]. From an economic perspective, modeling results of collaborative approaches reveal cost savings ranging from less than $10 \%$ [5], between 20 and 30\% [6-8] to as high as $50 \%$ [9]. The effectiveness of collaborative approaches can be influenced by various factors such as the geography [10] and not least the partners' similarity in distribution networks [11]. Only two freight carriers are participating in the research (demonstrator)

adfa, p. 1, 2011.

(C) Springer-Verlag Berlin Heidelberg 2011 
project but in a later implementation, a higher number of carriers are required to gain a high efficiency and to reduce the number of non-connecting deliveries.

From an environmental perspective, the reduction of empty backhaul trips was suggested as an alternative to mitigate the transport environmental footprint [10]. Environmental impact reductions will likely follow the cost reductions of collaborative approaches since fuel consumption accounts for a large proportion of transport costs.

Sustainability of the industrial sector has become one of the most significant societal, political and business issues due to the fact, that the manufacturing sector has a huge impact on the environment, economy and the quality of human life. The focus on the impact of supply chain activities including logistics and transportation has captured a huge academic and industrial interest which has led to significant contributions regarding figures and measures $[15,16]$, concepts and strategies and methodologies and tools such as Life-Cycle Assessment (LCA) and Corporate Social Responsibility (CSR).

While collaborative transport planning can generate a series of efficiency benefits, companies are also required to navigate challenges regarding information sharing and security with rivals and ensuring the delivery of adequate services by other companies for their own customers [4]. In cases where full information disclosure is accepted by collaborating parties, decisions can be made by a central decision-maker. However, in cases where not all information is shared amongst parties, decentralized decision-making approaches are adopted [2]. In a decentralized decision-making approach, transporters can retain the ability to choose which routes or cargoes they are willing to exchange. The decisions by individual transporters regarding routes or cargoes can depend on the information, options available [12], as well the relationships between logistics service providers and their customers [13, 14]. In this context, decision support systems can be useful tools to inform decision-makers of available collaboration alternatives while allowing the option for human direction of the final choice.

Today, only few companies are part of supply chains that jointly supports an overall planning body to optimize the flow. To support optimized goods flows between independent second-party logistics providers (2PLs) calls for supply chain collaboration solutions enabling increasing visibility and decision support integrated in current transport management systems. Firms are used to optimize internally, but collaborative logistics creates the need to develop a new business model that takes inter-firm relations into account.

This paper lines up the findings so far as well as conceptual solutions to support sharing of loads. Two options have been identified: an agent-based approach and an optimisation approach. The paper is organised as follows. First, the planning approach in the transport companies are presented alongside constraints in terms of policies (as well as driving regulations). Secondly, the identified options are presented followed by a discussion and conclusions. 


\section{$2 \quad$ Planning approach in case companies.}

The freight carriers in focus are specialised in handling point-to-point transport freights, normally minimum 5 pallets. The direct approach has a number of advantages compared to a hub-and-spoke setup that are mainly used for smaller freights. The main advantage is less handling and faster delivery time, whereas the downside is a higher risk of empty running. The freight carriers have three types of shippers: contractual, regular and occasional customers. Contractual customers have to be served, whereas regular customers could be turned down. Occasional customers are accepted if the load fits with the planned route and loads.

Most order requests come from customers, but some requests come from other transport companies aiming to sell of transports that do not fit into their routes and capacity. The actual load per day for contractual customers are not known until a few hours before the truck starts its route and may change over the day. This setup generates some uncertainty with respect to which transport orders to accept. On the one hand, if too many orders are accepted, the transport capacity may be insufficient for the day's deliveries. On the other hand, accepting fewer orders or a more risk-averse approach may mean that transport capacity is not fully utilized.

Due to uneven load balances, trucks are rarely fully loaded on both outbound and inbound trips. The goal is to have a full outbound load and aim for as high load as possible on the inbound trips. Most freight carriers have some casual transports to increase loads when possible, e.g. empty pallets. Other options is transport portals such as Timocom and Teleroute $[17,18,19]$ that act as a broker in between shippers and freight carriers. Uber Freight [20] is also entering the European market, but here the portal is a freight marketplace focused on full load transportation.

The transport companies investigated, plans their transports manually but use domain-specific systems to support the decision making and to share the current plan with colleagues as well as the final launch of trips. Orders from contractual customers arrive in semi-automated ways such as excel sheets or through manual access to customers information system. Regular and occasional customers communicate via email or phone. Surprisingly, very limited planning was conducted in real-time and re-filling of trucks were rarely used. The companies were aware that a further investigation of these areas could improve capacity utilisation and earnings.

The normal strategy by the route planners is to accept most requests as "it is easier to form a good plan if you have more orders". During the planning process, you may sell off requests that were accepted earlier in the planning process or contractual transports if the load either exceeds the transport capacity or is too small to fill up a truck. In the latter case, the planners contact transport colleagues by phone or email to sell of transports. Usually, the price of a transport increases during the day and therefore there is a risk element in having too many unallocated transports in the late afternoon. 


\section{$3 \quad$ Planning concepts.}

As previously stated, the idea with the paper is to present planning concepts supporting the current processes in transport companies. Two different concepts have been brought forward with the aim of improving the capacity utilisation:

- an agent-based approach aiming at supporting selling and buying transports to and from other transport companies

- an optimisation approach where all transports are re-distributed among the participating transport companies

under a given set of constraints configured by each participant such as:

- accepted transport colleagues to undertake a transport for the given company

- blocked customers and types of goods that are not to be transferred to colleagues

- a matrix expressing how may extra miles of route deviation you are willing to drive in order to pick up $\mathrm{X}$ number of pallets (e.g. 2 miles for 2 pallets and 20 miles for 10 pallets

- A threshold value expressing the minimum profit margin for accepting a request

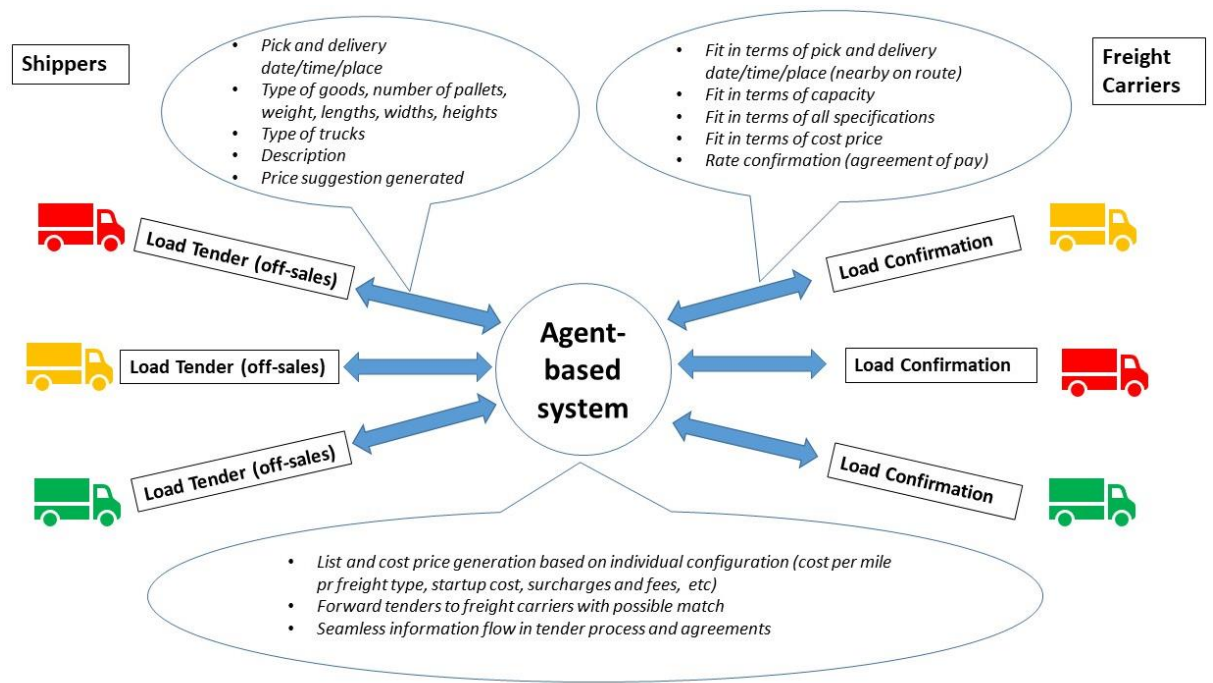

Fig. 1. Conceptual illustration of tenders, requests, constraints and business model

The constraints are used to evaluate as to whether a request from a customer or a transport company qualifies for manual decision making. To enable this pre-qualification process, the order request must as a minimum include the following information: type of goods, number of pallets, weight and volume, order date \& time, pick-up information (date \& time, address, customer name), delivery information (date \& time, address, customer name), and offered price. 
In an agent-based setup, the route planner will receive the pre-qualified requests which match the configured settings. This ensures that only relevant requests require the attention of the planner. Further, the system should support a full business process transaction in terms of automatically enter the order specification, notify the cargo owner when the product are delivered, invoice the cargo owner, etc. These processes are quite labour intensive in the current manual based setting.

As trucks constantly move and new orders are accepted while some old orders are sold off the picture changes over time. We have tried to illustrate this in figure 2 . The planning process is similar to the "Net-Change" in Enterprise Resource Planning (ERP) systems. This mean that during the day new orders are received and accepted and each time we consider how to best fit the new order in the existing plan. The alternative to this is a "Full plan" (in ERP this is done during the night or in some companies over the weekend due to time constraints) where all plans are cleared and a totally new plan is developed. The benefits of this is that the Net-Change does not find an optimal plan but a feasible plan given the already planned tasks.

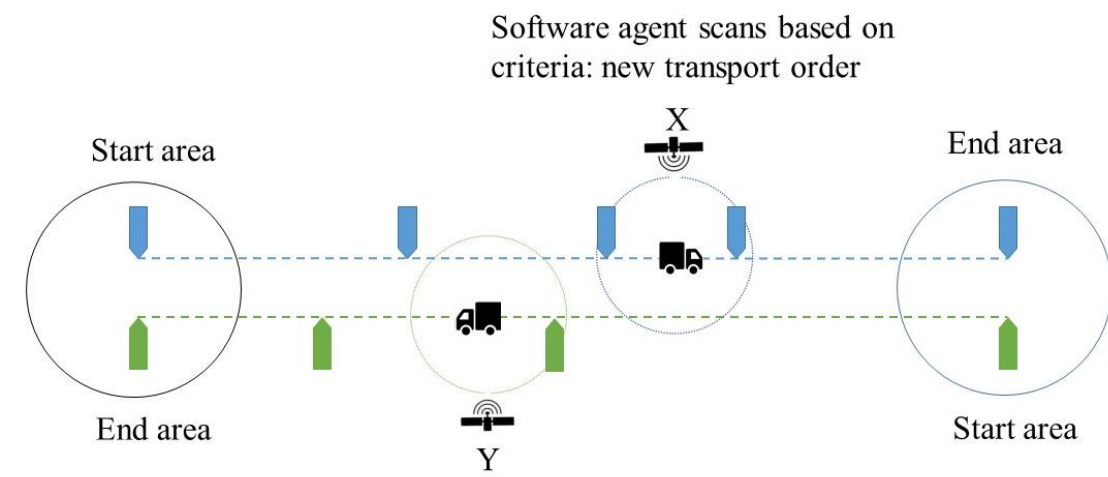

Software agent scans based on criteria: new transport order
X, Y: Criteria for scan
Domain partner 1
Domain partner 2

Fig. 2. Real-time scan for relevant tenders to accept or reject a request based on constraints such as position and time-window.

As an alternative to the agent-based planning concept a constrained or optimised concept could be considered. Constrained based planning is based on hard and soft (or goal) constraints [19]. Its distinguishing feature is that the objectives can be stated as minimising deviations from pre-specified goals. Hard constraints are not overruled, whereas soft constraints can be overruled, if necessary. If the number of trucks is considered as a hard constraint then capacity load, based on customer order acceptance, is considered as a soft constraint. As no plan optimisation objectives or criteria are considered, this option produces a feasible but not necessarily an optimal plan. Therefore, 
a hidden plan objective function is used to drive the planning and trade-off among the soft constraints. The hidden plan objective function is defined as minimizing plan cost. In addition to hard and soft constraints, it is possible to use business rules and demand priorities. Business rules are used as explicit decisions made when there are more options to choose among in the plan generation. Business rules are ranked by use of priorities of given topics and play an important role in constraint-based planning by avoiding the traditional (time-consuming) re-planning and re-scheduling after plan generation.

Optimised plans are generated based on plan objectives, penalty factors and constraints beside the hard and soft constraints [21]. The constraint-based rules are exchanged with decision variables and penalty factors, instead of the hidden object function and business rules and demand priorities. In the optimisation, the soft constraints might be overruled if this reduces the total costs. For example, demand priority and supplier allocation ranks could be overruled to reach the best profit.

The benefits of a constrained or optimised plan is that it automates the planning process. The question is, however, whether planning automation and elimination of individual decision-making for each company is a change that transport companies are willing to instantiate. With reference to the manufacturing area, it is a long "travel" to move from manual planning to semi-automated and fully automated planning, as this change requires reliable data and fit-for-purpose decision rules.

\section{Discussion and conclusion}

The paper discuss possible planning concepts in an automated collaborative logistics system based on real-time collection and analysis of shipment and tracking data which makes it possible for large competing logistics companies to share load capacity on less-than-truckload shipments and minimize empty-running. Currently, planning and coordination is handled manually and the aim of the ongoing project is to optimize the utilization of load capacity and minimize empty running, reducing costs, traffic congestion, and truck emissions.

At present only the most obvious constraints have been included in the work. Currently, we are e.g. not able to handle dependent options where one option only is relevant if another option also is fulfilled. It is also required that all trips have been initiated before the collaborative tool is able to suggest relevant loads to share (the chicken and egg challenge). Regular (returning) customers has less integrated planning routines but expect anyway that their loads are handled in spite of lacking contractual agreements. To support this, we suggest that historical data are used to forecast capacity requirements on a daily basis to make room for these customers.

Finally, a threshold value for the minimum number of empty pallets in the truck are needed if refill of trucks is considered. This value depends on the type of truck and the number of deliveries to be completed. The important issue here is to avoid unloading of many "new" pallets to enable offloading of "older" pallets. This last issue are to some degree in contrast to the initiating goal of obtaining a higher capacity load on trucks. 


\section{Acknowledgements.}

The authors would like to express their gratitude to the Innovation Fund Denmark which the Collaborative Cargo (Directly) research project, is a part off.

\section{References.}

1. Juan, A A; Faulin, J; Pérez-Bernabeu, E \& Jozafowiez, N (2014): "Horizontal cooperation in Vehicle Routing Problems with Backhauling anf Environmental Criteria. Procdia, Solial \& Behavioral Sciences, Vol 111 p 1133-1141

2 Gansterer, M., Hartl, R.F.: Collaborative vehicle routing: A survey. Eur. J. Oper. Res. 268, 1-12 (2018).

3. Cruijssen, F., Cools, M., Dullaert, W.: Horizontal cooperation in logistics: Opportunities and impediments. Transp. Res. Part E Logist. Transp. Rev. 43, 129-142 (2007).

4. Cruijssen, F.C.A.M.: Horizontal Cooperation in Transport and Logistics. CentER, Cent. Econ. Res. 46, 216 (2006).

5. Quintero-Araujo, C. L., Gruler, A., Juan, A.A.: Quantifying potential benefits of horizontal cooperation in urban transportation under uncertainty: A simheuristic approach. In: Conference of the Spanish Association for Artificial Intelligence. pp. 280-289 (2016).

6. Soysal, M., Bloemhof-Ruwaard, J.M., Haijema, R., van der Vorst, J.G.: Modeling a green inventory routing problem for perishable products with horizontal collaboration. Computers \& Operations Research. 89, 168-182 (2018).

7. Montoya-Torres, J.R., Muñoz-Villamizar, A., Vega-Mejia, C.A.: On the impact of collaborative strategies for goods delivery in city logistics. Prod. Plan. Control. 27, 443-455 (2016).

8. Bailey, E., Unnikrishnan, A., Lin, D.-Y.: Models forminimizing backhaul costs through freight collaboration. Journal of Transport Res. Board. 2224, 51-60 (2011).

9. Sanchez, M., Pradenas, L., Deschamps, J.-C., Parada, V.: Reducing the carbon footprint in a vehicle routing problem by pooling resources from different companies. NETNOMICS Economic Research and Electronic Networking. 17, 29-45 (2016).

10. Pérez-Bernabeu, E., Juan, A.A., Faulin, J., Barrios, B.B.: Horizontal cooperation in road transportation: A case illustrating savings in distances and greenhouse gas emissions. International Transactions in Operational Research. 22, 585-606 (2015).

11. Adenso-Díaz, B; Lozano, S; Moreno, P: Analysis of the synergies of merging multi-company transportation needs. Transportmetrica A: Transport Science. 10, 533-547 (2014).

12. Ukovich, W; Nolich, M; Fanti, M P; Iacobellis, G; Rusich, A: A Decision Support System for Cooperative Logistics. IEEE Trans. Autom. Sci. Eng. 14, 
732-744 (2017).

13. Gligor, D.M., Autry, C.W.: The Role of Personal Ralationships in Facilitating Supply Chain Communications: A Qualitative Study. J. Supply Chain Manag. 48, 24-43 (2012).

14. Gligor, D.M., Holcomb, M.: The role of personal relationships in supply chains: An exploration of buyers and suppliers of logistics services. Int. J. Logist. Manag. 24, 328-355 (2013).

15 Dornfeld, D.A. (2013): Green Manufacturing: Fundamentals and Applications, Springer, New York.

16 Bunse, K; Vodicka, M; Schönsleben, P; Brülhart, M \& Ernst, F (2011). “Integrating energy efficiency performance in production management - gap analysis between industrial needs and scientific literature, Journal of Cleaner Production, Vol. 19, Issues 6-7, pp 667-679

17 Timocom, www.timocom.com, last accessed April 12, 2019

18 Teleroute, www.teleroute.com, last accessed April 12, 2019

19 Trucker Path, truckerpath.com, last accessed April 12, 2019

20 Uber freight, www.uberfreight.com, last accessed June 9, 2019

21 Hooker, J. N, (2000), "Logic-Based Methods for Optimization: Combining Optimization and Constraint Satisfaction”. John Wiley \& Sons, New York. 